CECS-PHY-08/10

\title{
General Relativity with small cosmological constant from spontaneous compactification of Lovelock theory in vacuum
}

\author{
Fabrizio Canfora ${ }^{1}$, Alex Giacomini ${ }^{2,1}$, Ricardo Troncoso ${ }^{1,3}$ and Steven Willison* \\ ${ }^{1}$ Centro de Estudios Científicos, Casilla 1469, Valdivia, Chile. \\ ${ }^{2}$ Instituto de Física, Facultad de Ciencias, \\ Universidad Austral de Chile, Valdivia, Chile and \\ ${ }^{3}$ Centro de Ingeniería de la Innovación del CECS (CIN), Valdivia, Chile.
}

\begin{abstract}
It is shown that Einstein gravity in four dimensions with small cosmological constant and small extra dimensions can be obtained by spontaneous compactification of Lovelock gravity in vacuum. Assuming that the extra dimensions are compact spaces of constant curvature, General Relativity is recovered within certain class of Lovelock theories possessing necessarily cubic or higher order terms in curvature. This bounds the higher dimension to be at least seven. Remarkably, the effective gauge coupling and Newton constant in four dimensions are not proportional to the gravitational constant in higher dimensions, but shifted with respect to their standard values. This effect opens up new scenarios where a maximally symmetric solution in higher dimensions could decay into the compactified spacetime either by tunneling or through a gravitational analogue of ghost condensation. Indeed, this is what occurs requiring both the extra dimensions and the fourdimensional cosmological constant to be small.
\end{abstract}

PACS numbers: 04.50.-h, 04.50.Cd, 04.50.Kd

*Electronic address: canfora, giacomini, troncoso, steve@cecs.cl 


\section{INTRODUCTION}

The original dream of unifying the fundamental interactions through the dimensional reduction of pure General Relativity (GR) with cosmological constant in vacuum is appealing not least because of its simplicity and economy of ingredients. However, requiring both the inclusion of nonabelian fields and a small four-dimensional cosmological constant, is inconsistent with the fact that the compact "internal" manifold has to be of sufficiently small size (see e.g., [1] ). Thus, if one keeps GR as the higher-dimensional theory to begin with, one cannot avoid introducing new fundamental matter fields in higher dimensions. Albeit that this fact somehow perverts the original dogma of obtaining bosonic fields from pure geometry in higher dimensions, matter fields of a higher-dimensional origin could be welcome in this vein insofar as the arbitrariness in their choice were removed by requiring some basic principle for the fundamental theory to hold; as for instance, local supersymmetry. Most of what we have learned about dimensional reduction has been developed along this line, mainly within the context of supergravity in diverse dimensions and string theory (For a review see, e.g., [2] and references therein).

We propose to explore whether one could return to the original dream of having a realistic model for bosonic fields arising from pure geometry in higher dimensions. This scenario should then accommodate consistently nonabelian gauge fields and small cosmological constant with a sufficiently small compact internal manifold.

Note that in dimensions higher than four, GR is not the only option for the gravity theory to begin with. Indeed, the same basic requirements that yield the Einstein-Hilbert action with cosmological constant in four dimensions, i.e., general covariance and second order field equations for the metric, give rise to the Lovelock action [3]. This action contains higher powers in the curvature in a precise combination and its Kaluza-Klein reduction will always give field equations for the matter fields of second order. The simplest modification of GR in higher dimensions corresponds to the addition of quadratic terms in the so-called GaussBonnet combination. Thus, it is natural wondering whether the Einstein-Gauss-Bonnet (EGB) theory admits suitable spontaneous compactifications to four dimensions. In the case of spontaneous compactifications of EGB theory in vacuum, the Einstein equations are recovered, but with an additional scalar condition which overconstrains the four-dimensional gravitational field, so that even the Schwarzschild solution becomes excluded [4]. 
Nonetheless, here it is shown that GR with small cosmological constant, without any additional constraint on the four-dimensional geometry, can be recovered from spontaneous compactifications with small extra dimensions, provided cubic or higher order terms in the Lovelock series are considered, with a single relation amongst the coupling constants. Remarkably, the effective four-dimensional Newton constant is not proportional to the gravitational constant in higher dimensions, but instead shifted with respect to the standard value. This effect opens up new interesting scenarios which are analyzed below. The mechanism bounds the higher dimension to be at least seven, since this is the smallest dimension that admits a nontrivial cubic term in the action. It is instructive to perform the complete analysis in this case.

\section{SPONTANEOUS COMPACTIFICATIONS.}

In seven dimensions, the Lovelock action acquires a simple expression when it is written in terms of differential forms, which reads

$$
\begin{aligned}
I_{7} & =\int_{M_{7}} \epsilon_{A B C D E F G}\left(c_{3} R^{A B} R^{C D} R^{E F}+\frac{c_{2}}{3} R^{A B} R^{C D} e^{E} e^{F}\right. \\
& \left.+\frac{c_{1}}{5} R^{A B} e^{C} e^{D} e^{E} e^{F}+\frac{c_{0}}{7} e^{A} e^{B} e^{C} e^{D} e^{E} e^{F}\right) e^{G},
\end{aligned}
$$

where $R^{A B}$ and $e^{A}$ stand for the curvature two-form and the vielbein, respectively, and wedge product between forms is understood. Let us look for spontaneous compactifications of the form $M_{7}=M_{4} \times K_{3}$, where $M_{4}$ is a four-dimensional Lorentzian manifold, and $K_{3}$ is a compact Euclidean manifold, which for simplicity is assumed to be of constant curvature $R^{a b}=\Lambda_{3} e^{a} e^{b}$. Indices are split into greek on $M_{4}$ and lowercase Latin on $K_{3}$. Then, it is simple to show that in the vanishing torsion sector, the field equations along $M_{4}$ reduce to the Einstein equations with cosmological constant in vacuum

$$
\frac{1}{8 \pi \tilde{G}_{4}}\left(G_{\mu \nu}+\Lambda_{4} g_{\mu \nu}\right)=0
$$

where the effect of the quadratic and cubic terms just amounts to a redefinition of the Newton and cosmological constants, given by $\tilde{\kappa}_{4}:=\left(16 \pi \tilde{G}_{4}\right)^{-1}=4 ! \operatorname{Vol}\left(K_{3}\right)\left(c_{1}+c_{2} \Lambda_{3}\right)$ and $\Lambda_{4}=-3 \frac{5 c_{0}+c_{1} \Lambda_{3}}{c_{1}+c_{2} \Lambda_{3}}$. Here, $\operatorname{Vol}\left(K_{3}\right)$ stands for the volume of $K_{3}$. Then, the remaining field equations, corresponding to the projection along $K_{3}$, generically reduce to an additional 
scalar condition on the four-dimensional geometry, fixing the Euler density of $M_{4}$ to be a constant, i.e.,

$$
\begin{gathered}
\left(c_{2}+3 c_{3} \Lambda_{3}\right)\left(R^{\mu \nu \lambda \rho} R_{\mu \nu \lambda \rho}-4 R^{\mu \nu} R_{\mu \nu}+R^{2}\right)+ \\
24\left[\frac{c_{0}\left(5 \Lambda_{3} c_{2}-15 c_{1}\right)-\Lambda_{3} c_{1}\left(5 c_{1}-\Lambda_{3} c_{2}\right)}{c_{1}+c_{2} \Lambda_{3}}\right]=0 .
\end{gathered}
$$

This equation conflicts with most solutions of the Einstein equations. In particular, for the EGB theory $\left(c_{3}=0\right)$ the four-dimensional gravitational field becomes overconstrained, so that even the Schwarzschild-(A)dS solution is excluded. In the presence of (3), spontaneous compactifications have been found [5]. However, the obstruction on the four-dimensional metric imposed by (3) can be eliminated when the cubic term is switched on, provided the curvature radius of $K_{3}$ is fixed as $\Lambda_{3}=-c_{2} /\left(3 c_{3}\right)$, for the class of theories whose couplings are related such that the square bracket of (3) vanishes. Since the cubic term must be present in order for the above mechanism to work, the coupling $c_{3}$ can be regarded as the overall factor of the action, so that it is useful to work with the rescaled couplings $\tilde{c}_{i}=c_{i} / c_{3}$.

In sum, the class of theories given by

$$
\tilde{c}_{0}=\frac{\tilde{c}_{1} \tilde{c}_{2}\left(15 \tilde{c}_{1}-\tilde{c}_{2}^{2}\right)}{15\left(9 \tilde{c}_{1}+\tilde{c}_{2}^{2}\right)}
$$

admits spontaneous compactifications in vacuum where the four-dimensional geometry fulfills the Einstein field equations in vacuum with neither corrections nor further constraints, and the effect of the quadratic and cubic terms just amounts to a redefinition of the Newton and cosmological constants, which are given by

$$
\tilde{\kappa}_{4}=4 ! \operatorname{Vol}\left(K_{3}\right) c_{3}\left(\tilde{c}_{1}-\frac{\tilde{c}_{2}^{2}}{3}\right) ; \Lambda_{4}=-\frac{6 \tilde{c}_{1} \tilde{c}_{2}}{9 \tilde{c}_{1}+\tilde{c}_{2}^{2}},
$$

respectively, and the curvature radius of the extra dimensions turns out to be fixed only in terms of the rescaled Gauss-Bonnet coupling, $\Lambda_{3}=-\tilde{c}_{2} / 3$.

This clearly differs from the spontaneous compactification of the Einstein theory which fixes both, $\Lambda_{3}$ and $\Lambda_{4}$ to be proportional to the cosmological constant in seven dimensions, preventing the compatibility of a tiny four-dimensional cosmological constant with small enough extra dimensions. Remarkably, the obstruction appearing in GR can be surmounted in the present framework, since the induced cosmological constant in four dimensions (5) depends on two parameters. Hence, in this scenario it is possible to accommodate consistently a tiny cosmological constant in four dimensions with a sufficiently small compact internal 
manifold. The condition $\left|\Lambda_{4} / \Lambda_{3}\right| \ll 1$ amounts to requiring $\left|9 \tilde{c}_{1} / \tilde{c}_{2}^{2}\right| \ll 1$, from which it is apparent that the higher curvature terms cannot be regarded as small corrections of GR in higher dimensions. Note that it is also possible to have $\Lambda_{3}$ and $\Lambda_{4}$ of opposite signs without the need of introducing matter fields.

Note that, the effective four-dimensional Newton constant $\tilde{\kappa}_{4}$ in Eq. (5) acquires a shift as compared with the standard value $\kappa_{4}$ obtained from Einstein's theory, which in our conventions reads $\kappa_{4}=4 ! \operatorname{Vol}\left(K_{3}\right) c_{1}$. Remarkably, the positivity of $\tilde{\kappa}_{4}$ can be guaranteed even if the standard Newton constant in higher dimensions, given by $c_{1}=\left(4 ! 16 \pi G_{7}\right)^{-1}$, is negative or even vanishing. In other words, it is possible to recover GR in four dimensions from a seven-dimensional theory even without Einstein-Hilbert term. This effect opens up new interesting scenarios where a maximally symmetric solution in higher dimensions could decay into the spontaneously compactified spacetime either by tunneling or through a gravitational analogue of ghost condensation. Indeed, we now show that this is what occurs when both the extra dimensions and the four-dimensional cosmological constant are required to be to be small.

\section{MAXIMALLY SYMMETRIC SPACETIMES VERSUS SPONTANEOUS COMPACTIFICATIONS.}

The presence of cubic terms in the action (11) allows the existence of up to three maximally symmetric solutions, and each of them, in principle, can be regarded as an uncompactified ground state. Indeed, in the case of constant curvature spacetimes, $R^{A B}=\lambda_{7} e^{A} e^{B}$, the field equations reduce to a cubic polynomial in $\lambda_{7}$ given by

$$
P\left(\lambda_{7}\right):=\lambda_{7}^{3}+\tilde{c}_{2} \lambda_{7}^{2}+\tilde{c}_{1} \lambda_{7}+\tilde{c}_{0}=0
$$

and hence the theory admits at most three possible maximally symmetric ground states whose radii are determined by the real roots $\bar{\lambda}_{7}$ of ([6]) .

Note that generically, the seven-dimensional Newton constant is not determined by the coefficient $c_{1}$ in front of the Einstein-Hilbert term, which could even be absent from the very beginning, since the linearized field equations around any of the maximally symmetric ground states of curvature $\bar{\lambda}_{7}$, reduce to the standard Fierz-Pauli equation but with a different overall factor. This can be seen linearizing the field equations, expressing the vielbein as 
$e^{A}=\bar{e}^{A}+\delta e^{A}$, where $\bar{e}^{A}$ stands for the chosen background. The linearized field equations then read

$$
c_{3} P^{\prime}\left(\bar{\lambda}_{7}\right) \epsilon_{A_{1} \ldots A_{6} B} \delta\left(R^{A_{1} A_{2}}-\bar{\lambda}_{7} e^{A_{1}} e^{A_{2}}\right) \bar{e}^{A_{3}} \ldots \bar{e}^{A_{6}}=0,
$$

and the corresponding Newton constant is given by

$$
\bar{\kappa}_{7}:=\frac{1}{16 \pi \bar{G}_{7}}=4 ! c_{3} P^{\prime}\left(\bar{\lambda}_{7}\right) .
$$

Hence, each ground state possesses its own Newton constant that depends on $c_{3}$ and the slope of the polynomial (6) evaluated at the corresponding root. Thus, the linearized field equations acquire support provided the expansion is performed around a nondegenerate root. Since the sign of the second variation of the effective action that defines the graviton propagator is determined by $\bar{\kappa}_{7}$, for different uncompactified ground states, the graviton could behave as a particle or as a ghost, as occurs for the EGB theory [6].

It is worth remarking that the maximally symmetric spacetimes for which the graviton behaves as a ghost should not be discarded from scratch. Indeed, from the point of view of dimensional reduction, one might expect that they correspond to a sort of false vacua which could decay to the spontaneously compactified spacetime, where the graviton has a well-defined propagator, through a gravitational analogue of ghost condensation (see Ref. [7]). In this case, the transition, instead of being driven by an additional scalar field, is triggered by the higher dimensional graviton in vacuum. Note that even those vacua whose seven-dimensional graviton does behave as a particle could still be metastable against decay into the compactified spacetime by tunneling.

Both possibilities open up new interesting scenarios which do not occur for spontaneous compactifications of (super) gravity based on the Einstein-Hilbert action in higher dimensions, which nevertheless could be compatible with some more recent alternatives to compactification [8]. Note that for a theory that possesses a maximally (super)symmetric vacuum with well-defined propagators around it, it is not simple to explain why the theory does not prefer this configuration as the ground state, instead of choosing a compactified spacetime. In the present framework, considering a theory that does not possesses a suitable uncompactified ground state is welcome, since it could naturally provide a dynamical mechanism of spontaneous dimensional reduction (see also [9]).

Let us discuss the possible scenarios within the class of theories defined by (44), which admits spontaneous compactifications consistent with GR in vacuum. The arbitrariness in 
the choice of the coupling constants for this class of theories is further restricted requiring a few conditions compatible with a realistic four-dimensional picture. Thus, requiring both a tiny (preferably positive) four-dimensional cosmological constant and small enough extra dimensions implies $\left|\Lambda_{4}\right| \ll\left|\Lambda_{3}\right|$, which in terms of the coupling constants reads $9\left|\tilde{c}_{1}\right| \ll \tilde{c}_{2}^{2}$. Consequently, since the effective Newton constant in four dimensions $\tilde{\kappa}_{4}$ has to be positive, Eq. (5) implies that the overall factor in front of the action must be negative. In other words, the condition $c_{3}<0$ is necessary in order to have a well-defined propagator for the graviton in four dimensions.

Albeit it is not necessary, hereafter we restrict to the case $\Lambda_{3}>0$ (i.e. $\tilde{c}_{2}<0$ ) in order to allow the presence of non-Abelian gauge fields. Note that $c_{2}>0$ is compatible with string theory [6]. The analysis then splits into three cases according to the sign of $\tilde{c}_{1}$, which due to the above requirements, coincides with the sign of $\Lambda_{4}$ :

$\Lambda_{4}<0$ : The polynomial (6) has positive slope at its unique real root which is simple and positive. Thus, there is a single maximally symmetric solution of positive curvature $\left(d S_{7}\right)$, and since $\bar{\kappa}_{7}<0$ (see Eq. (7)), the seven-dimensional graviton behaves like a ghost. The spontaneous compactification has a well-defined graviton in four dimensions (see Eq. (5)). This suggests that $d S_{7}$ could spontaneously decay into the less symmetric solution, $A d S_{4} \times S^{3}$, through the gravitational analogue of ghost condensation.

$\Lambda_{4}=0$ : The polynomial $P\left(\lambda_{7}\right)$ has two real roots, and it has positive slope at the simple one which is positive. Thus, one of the maximally symmetric vacua corresponds to $d S_{7}$, which is expected to decay through gravitational ghost condensation into the compactified vacuum, $\mathbb{M}_{4} \times S^{3}$, having a well-defined propagator for the graviton. The doubly degenerate root vanishes (i.e., $\bar{\lambda}_{7}=0$ and $\bar{\kappa}_{7}=0$ ), so that the maximally symmetric solution is Minkowski spacetime around which the linearized equation for graviton has no support. Thus, since the propagator is ill-defined, flat spacetime is expected to be a sort of false vacuum. This scenario is similar to the one in Ref. [9], where it is shown that in order to have propagation for the graviton, the spatial components of the curvature cannot be small. Hence, propagating deviations around flat spacetime are nonlocal and would require too much energy, since they are nonperturbative. In this case the spontaneous compactified spacetime, $\mathbb{M}_{4} \times S^{3}$, is expected to be preferred since it admits a well-defined low energy limit.

$\Lambda_{4}>0: P\left(\lambda_{7}\right)$ has three real roots, $\lambda_{7}^{(1)}<0<\lambda_{7}^{(2)}<\lambda_{7}^{(3)}$. As the slope of the polynomial at $\lambda_{7}^{(1)}$ and $\lambda_{7}^{(3)}$ is positive, the seven-dimensional graviton behaves as a ghost around the 
corresponding maximally symmetric $A d S_{7}$ and $d S_{7}$ spacetimes, respectively, which would decay into the spontaneous compactification $d S_{4} \times S^{3}$. Since the slope of the polynomial at the remaining root is negative, the corresponding Newton constant becomes positive, and so the graviton in seven dimensions acquires a well-defined propagator around $d S_{7}$ with curvature $\lambda_{7}^{(2)}$. Nonetheless, this seven-dimensional vacuum appears to be metastable against decay through tunneling into the compactified configuration $d S_{4} \times S^{3}$. A crude estimate of the tunneling rate can be obtained in the semiclassical approximation through the difference of the Euclidean version of the action (11) evaluated on the corresponding Eucildean continuations, $S^{7}$ and $S^{4} \times S^{3}$, respectively.

In the case of $S^{4} \times S^{3}$, for theories with $x:=9 \tilde{c}_{1} \tilde{c}_{2}^{-2}=\left(2 \Lambda_{3} / \Lambda_{4}-1\right)^{-1} \ll 1$ (i.e., $\left.\Lambda_{4} \ll \Lambda_{3}\right)$, the Euclidean action, up to an overall positive factor, becomes $I_{4,3} \simeq-c_{3} x^{-1}$; while for $S^{7}$, of curvature given by $\lambda_{7}^{(2)}=\left(\tilde{c}_{1} / 15\right)^{1 / 2}+\mathcal{O}(x)$, the action is given by $I_{7} \simeq-c_{3} x^{-3 / 4}$. Since in our conventions the most likely vacuum is the one with the larger Euclidean action, for a realistic picture (i.e. for small $x$ ), the seven-dimensional de Sitter solution turns out to be metastable against tunneling into the spontaneously compactified vacuum $d S_{4} \times S^{3}$, because the latter clearly has a larger Euclidean action.

Note that this is not a possibility for the Einstein theory with positive $\Lambda_{7}$ in vacuum, since not only $\Lambda_{3}$ and $\Lambda_{4}$ are of the same order as $\Lambda_{7}$, but the tunneling mechanism goes in the opposite way, since $d S_{7}$ turns out to be more likely than $d S_{4} \times S^{3}$. Indeed, as the on-shell value of the Euclidean action is given by $I_{E H}\left(M_{7}\right)=\left(20 \pi G_{7}\right)^{-1} \Lambda_{7} \operatorname{Vol}\left(M_{7}\right)$, and as the seven sphere has a better distributed volume than $S^{4} \times S^{3}$, one obtains that $I_{E H}\left(S^{7}\right) / I_{E H}\left(S^{4} \times S^{3}\right)=3^{3 / 2} / 4>1$. Therefore, GR in vacuum prefers the uncompactified spacetime. In our framework as the action possesses additional fundamental constants, the tunneling mechanism acquires a control parameter $x$, which for a small enough value, allows a concrete realization of spontaneous breakdown of the vacuum symmetry from $S O(7,1)$ to $S O(4,1) \times S O(4)$.

In sum, for the class of theories admitting spontaneous compactifications consistently with GR in vacuum, defined by (4), once required to be compatible with a reasonable fourdimensional picture, the spontaneously compactified vacuum turns out to be preferred with respect to the maximally symmetric spacetime, since the latter would decay either by the gravitational analogue of ghost condensation or by tunneling. 


\section{GAUGE FIELDS WITH SHIFTED COUPLING.}

Since the theory (1) is generally covariant the massless gauge fields are guaranteed to be gauge invariant. Switching on only the massless modes for the gauge field, the field equations reduce to Yang-Mills at the linearized level, i.e.

$$
4\left(3 c_{1}+c_{2} \Lambda_{3}\right) \bar{\nabla}^{\mu} \partial_{[\mu} A_{\nu]}^{(a)}=0
$$

Hence, as $\Lambda_{3}=-c_{2} /\left(3 c_{3}\right)$, the gauge coupling is shifted by $c_{1} \rightarrow c_{1}-c_{2}^{2} /\left(9 c_{3}\right)$, compared with Weinberg's formula obtained from compactification of GR [10]. Note that the gauge coupling is neither proportional to the standard nor the effective Newton constant (5). The gauge field possesses a well-defined propagator provided the effective gauge coupling is positive, and it is reassuring to verify that this is the case for the realistic scenarios discussed above with $\left|\Lambda_{4} / \Lambda_{3}\right| \ll 1$ and $\tilde{\kappa}_{4}>0$.

As occurs for the compactifications of GR, the analysis of the scalar field propagator requires special attention. This, as well as how the Kaluza-Klein tower differs from the one obtained from standard (super)gravity in higher dimensions, is left for future research. It is also worth mentioning that the fact that Lovelock theories admit propagating degrees of freedom for torsion in vacuum [11] may help to obtain realistic chiral fermions from spontaneous compactifications [12].

The mechanism that leads to spontaneous compactifications in vacuum consistent with GR in four dimensions actually carries through in $D \geq 7$ dimensions. It would be interesting to explore compactifications on product spaces, since as it occurs in standard supergravity, this may improve the stability of the solution, naturally suggesting time-dependent compactifications [13]. Previous results [14] indicate that within our framework the compact manifold may shrink in time (related cosmological models have been studied in [15]). For theories with quartic or higher powers of the curvature it is possible to have more than one possible compactification radius, enlarging the class of theories that admits suitable spontaneous compactifications. Note that, for theories with quartic or higher even powers of the curvature, a maximally symmetric vacuum may not even exist, suggesting that the ground state must be compactified. This is discussed in a forthcoming publication. 


\section{Acknowledgments}

We thank G. Giribet, G. Kunstatter, D. Marolf and J. Oliva for helpful comments. This work was partially funded by FONDECYT grants $\mathrm{N}^{o}$ 1051056, 1061291, 1071125, 1085322, 3070057, 11080056, 1095098 and UACH-DID grant No. S-2009-57. Centro de Estudios Científicos (CECS) is funded by the Chilean Government through the Millennium Science Initiative and the Centers of Excellence Base Financing Program of CONICYT. CECS is also supported by a group of private companies which at present includes Antofagasta Minerals, Arauco, Empresas CMPC, Indura, Naviera Ultragas and Telefónica del Sur. CIN is funded by CONICYT and Gobierno Regional de Los Ríos.

[1] J. M. Overduin and P. S. Wesson, Phys. Rept. 283, 303 (1997).

[2] M. R. Douglas and S. Kachru, Rev. Mod. Phys. 79, 733 (2007).

[3] D. Lovelock, J. Math. Phys. 12, 498 (1971).

[4] G. Giribet, J. Oliva and R. Troncoso, JHEP 0605, 007 (2006).

[5] F. Mueller-Hoissen, Phys. Lett. B 163, 106 (1985). H. Maeda and N. Dadhich, Phys. Rev. D 74, 021501 (2006). M. H. Dehghani and S. Assyyaee, arXiv:0811.0877 [gr-qc].

[6] D. G. Boulware and S. Deser, Phys. Rev. Lett. 55, 2656 (1985).

[7] N. Arkani-Hamed, H. C. Cheng, M. A. Luty and S. Mukohyama, JHEP 0405, 074 (2004).

[8] N. Arkani-Hamed, S. Dimopoulos and G. R. Dvali, Phys. Lett. B 429, 263 (1998). I. Antoniadis, N. Arkani-Hamed, S. Dimopoulos and G. R. Dvali, Phys. Lett. B 436, 257 (1998).L. Randall and R. Sundrum, Phys. Rev. Lett. 83, 4690 (1999).

[9] M. Hassaine, R. Troncoso and J. Zanelli, Phys. Lett. B 596, 132 (2004).

[10] S. Weinberg, Phys. Lett. B 125, 265 (1983).

[11] R. Troncoso and J. Zanelli, Class. Quant. Grav. 17, 4451 (2000).

[12] E. Witten, Nucl. Phys. B 186, 412 (1981).

[13] P. G. O. Freund and P. Oh, Nucl. Phys. B 255, 688 (1985); P. Oh, Phys. Lett. B 166, 292 (1986).

[14] N. Deruelle and L. Farina-Busto, Phys. Rev. D 41, 3696 (1990).

[15] N. Deruelle and J. Madore, Phys. Lett. B 186, 25 (1987); Phys. Lett. A 114, 185 (1986). 